\title{
A CASE OF NEUROFIBROMATOSIS TYPE 1
}

\author{
Valentina Dimitrova $^{1}$, Ivelina Yordanova ${ }^{1}$, Verka Pavlova ${ }^{1}$, Valentin Valtchev ${ }^{1}$, \\ Dimitar Gospodinov ${ }^{1}$, Boryana Parashkevova ${ }^{2}$, Chavdar Balabanov ${ }^{2}$ \\ ${ }^{1}$ Department of Dermatology and Venereology, \\ ${ }^{2}$ Department of Ophthalmology, \\ Medical University - Pleven, Bulgaria
}

\begin{abstract}
Neurofibromatosis (NF) is a term that has been applied to a variety of related syndromes, characterized by neuroectodermal tumors arising within multiple organs and autosomal-dominant inheritance. Neurofibromatosis type I (NF-1), known as well as Recklinghausen's disease, is the most common type of the disease accounting $90 \%$ of the cases. We present a case of 52-year-old men with NF-1. The disease started in childhood with the appearance of multiple hyperpigmented skin macules. At the age of 46 a lot of cutaneous tumors appeared and started growing bigger all over the body surface. Because of a vision problem due to an upper left eyelid neurofibroma, the patient came for a clinical examination at the age of 52 years.
\end{abstract}

Key words: Neurofibromatosis, Neurofibromatosis type I, Recklinghausen's disease

\section{INTRODUCTION}

Neurofibromatosis (NF) is a term that has been applied to a variety of related syndromes, characterized by neuroectodermal tumors arising within multiple organs and autosomal-dominant inheritance. At least 8 different clinical phenotypes of neurofibromatosis have been identified and are linked to at least two genetic disorders. Neurofibromatosis type I (NF-1) is the most common type of the disease accounting $90 \%$ of the cases, and is characterized by multiple cafй-au-lait spots and the occurrence of neurofibromas along peripheral nerves.

\section{CASE REPORT}

History

A 52-year-old man with Neurofibromatosis type I is presented. The disease started in childhood with the appearance of multiple hyperpigmented skin macules. At the age of 46 a lot of cutaneous tumors appeared and started to increase in size all over the body surface especially on the left eyelid. Due to his psychic condition (the patient is mentally retarded after encephalitis in childhood) he has not consulted a doctor. The growth of the fibroma on the upper eyelid of the left eye had caused visual difficulties, which made him seek care.

\section{Physical examination}

Dermatological status: hundreds of soft cutaneous neurofibromas, the largest amount being on the trunk and limbs, ranging from a few millimeters to several centimeters in diameter (fig 2), some of them pedunculated (fig 3); multiple cafŭ-au-lait spots with diameter $>1,5 \mathrm{~cm}$ (fig 4); axillary and inguinal freckling (fig 5). The mucous membranes were not affected.

Ophthalmological status: multiple cutaneous fibromas of different size on eyelids of both eyes (Fig. 6), without inflammation. There was a $1,5 \mathrm{~cm}$ fibroma that affected the edge of eyelid and spread approximately $1 \mathrm{~cm}$ to the eyelid margin on the lateral part of the upper eyelid of the left eye. The upper eyelid had partial secondary ptosis. Lisch's nodules on the iris of both eyes were without clinical visual involvement (fig 7).

\section{Lab and imaging studies, histologic findings and consultations}

The standard laboratory tests values were in the normal range. X-ray photography and $\mathrm{CT}$ are within the normal too. The neurologist did not detect alterations in the central and peripheral nervous system. According to the otologist the acoustic nerve has not been damaged. The histological result confirmed the diagnosis of Neurofibromatosis.

\section{DIAGNOSIS}

The diagnosis NF-1 was made according to the presence of four of the seven diagnostic criteria of the National Institute of Health Consensus Development Conference:

- Five or more cafe-au-lait spots larger than $5 \mathrm{~mm}$ in diameter in prepubertal patients; six or more cafŭ-au-lait spots larger than $15 \mathrm{~mm}$ in diameter in postpubertal patients

- Two or more neurofibromas of any type, or one plexiform neurofibroma

- Axillary or inguinal freckling

- Two or more Lisch's nodules

\section{Therapy}

Because of the restriction in the peripheral visual field of the left eye due to the large tumor on the upper eyelid, 
the patient was hospitalized in the Ophthalmologic clinic in Pleven for excision of the tumor, which was successful and achieved a good cosmetic result. The material left after excision was sent for histological examination. After the excision, the configuration of upper eyelid was restored and peripheral vision of left eye - which was the main complain of the patient - was improved.

\section{DISCUSSION}

Manifestations of neurofibromatosis have been observed for a long time before being described by Robert William Smith in $1849^{1}$. The classic description is by a German pathologist, Friedrich Daniel von Recklinghausen, who accurately described the diverse findings as a single entity in $1882^{2}$; thus the condition is often referred to as von Recklinghausen's disease (fig 1).

There is no single commonly accepted classification. According to the most widely accepted classification, there are four recognized forms of neurofibromatosis:

- von Recklinghausen's neurofibromatosis (or neurofibromatosis type 1 [NF-1] or peripheral neurofibromatosis)

- Bilateral acoustic neurofibromatosis (or neurofibromatosis type 2 [NF-2] or central neurofibromatosis)

- Segmental neurofibromatosis

- Cutaneous neurofibromatosis

Riccardi ${ }^{3}$ suggested the presence of three additional forms: type 3 (mixed), type 4 (variant) and type 5 (late-onset). However, these may not represent separate conditions.

The neurofibromatosis comprise of at least two separate genetic disorders (NF-1 and NF-2) characterized by the formation of tumours surrounding nerves and a variety of other pathological features. As many as six additional types have been proposed to characterize what appear to be clinically distinct conditions within this group.

The most common type (NF-1) accounting for $90 \%$ of cases, is characterized by multiple cafe-au-lait spots and the occurrence of neurofibromas along peripheral nerves. Cutaneous neurofibromas are soft, flesh- or lilac-pinkcoloured tumours, sessile or dome-shaped, sometimes pedunculated, and most numerous on the trunk and limbs. Other clinical features include Lisch's nodules (melanocytic pigmented iris hamartomas) and oral lesions. Possible complications in childhood include the development of an optic glioma, endocrine disturbances and involvement of the lower urinary tract. The children may also present with learning disabilities.

Von Recklinghausen's neurofibromatosis (NF-1) is inherited in an autosomal-dominant fashion and has a prevalence of between 1 per $3000^{4}$ and 1 per $5000^{5}$ live births thus being one of the most common autosomal-dominant conditions in humans. The penetrance of NF-1, or the proportion of people with the NF1 gene with a clinical presentation of the disorder, is close to $100 \%$ but because the mutation rate is so high, about a half of the newly diagnosed cases may represent with new mutations. The gene has been isolated to the proximal long arm of chromosome $17(17,11.2)$.

\section{Diagnostic Criteria}

According to the National Institute of Health Consensus Development Conference ${ }^{6}$, at least two of the following criteria must be present to make the diagnosis of NF-1:

1. Five or more cafe-au-lait spots larger than $5 \mathrm{~mm}$ in diameter in prepubertal patients; six or more cafe-au-lait spots larger than $15 \mathrm{~mm}$ in diameter in postpubertal patients

2. Two or more neurofibromas of any type, or one plexiform neurofibroma

3. Axillary or inguinal freckling

4. Optic glioma

5. Two or more Lisch's nodules

6. A distinctive osseous lesion (pseudoarthrosis of the tibia or sphenoid wing dysplasia)

7. A first-degree relative diagnosed with NF-1 in accordance with the above criteria

Plexiform neurofibromas of the orbit tend to originate from the orbital branches of the trigeminal nerve. They often affect the upper eyelid, causing a characteristic sinusoidal deformity of the lid margin. ${ }^{7}$ The tumor is soft and feels like a "bag of worms"; the resultant displacement of the globe or ptosis can result in amblyopia in children. Plexiform neuromas of the orbit are associated with congenital absence of the sphenoid or enlargement of the sella turcica. Peripheral neurofibromas are benign tumors consisting predominately of Schwann's cells and fibroblasts with endothelial, perineural, and mast cells. ${ }^{8}$ There is evidence that they have a single-cell origin despite multiple cell types within the tumors. ${ }^{9}$ Plexiform neurofibromas occur in about one third of NF-1 cases, most commonly on the trunk and less often on the limbs, head and neck. They are benign and rarely symptomatic, but they can cause significant cosmetic and visual problems if the orbit is involved. Саfйau-lait spots are composed of epidermal melanocytes with giant pigment granules (macromelanosomes) within the cytoplasm and are of neural crest origin. They are not pathognomonic of neurofibromatosis, having been reported in association with several other conditions and in patients not affected by the condition. ${ }^{10}$

Hamartomas of the iris (melanocytic nevi) can be seen and are called Lisch nodules. They are variable in size and have a smooth, dome-shaped configuration. ${ }^{11}$ One study found these nodules in $92 \%$ of the affected population over the age of 6 years; this may mean that their absence prior to that age does not rule out their later occurrence. Lisch nodules may also be seen in the trabecular meshwork. ${ }^{12}$ In a more recent study, the incidence of Lisch nodules in 
patients with neurofibromatosis beyond the second decade of life, was $100 \% \cdot{ }^{13}$ Lisch nodules, which can be indicative of Neurofibromatosis 1 when multiple, are rarely seen in Neurofibromatosis $2 .{ }^{14}$

Although clinical findings are primarily Neurocutaneous in nature, any organ system can be involved. The diagnosis requires six or more cafe au lait cafe-au-lait spots, each larger than $1,5 \mathrm{~cm}$ in diameter. Axillary freckling is also highly suggestive of the diagnosis. ${ }^{15,16}$ Areas of hypopigmentation or hyperpigmentation can also be seen.

\section{CONCLUSION}

The patient described here is a very typical case of NF-1, which presents a considerable interest because of the high generalization of the skin lesion. In such cases, a detailed patient investigation is required, because of the possibility for generalized involvement of other organs. The proper clinical and genealogic analysis is important for the determination of the genetic risk and prognosis for the relatives of the proband. The treatment of such kind of patient is surgical, seeking to achieve cosmetic improvement, and may be only palliative.
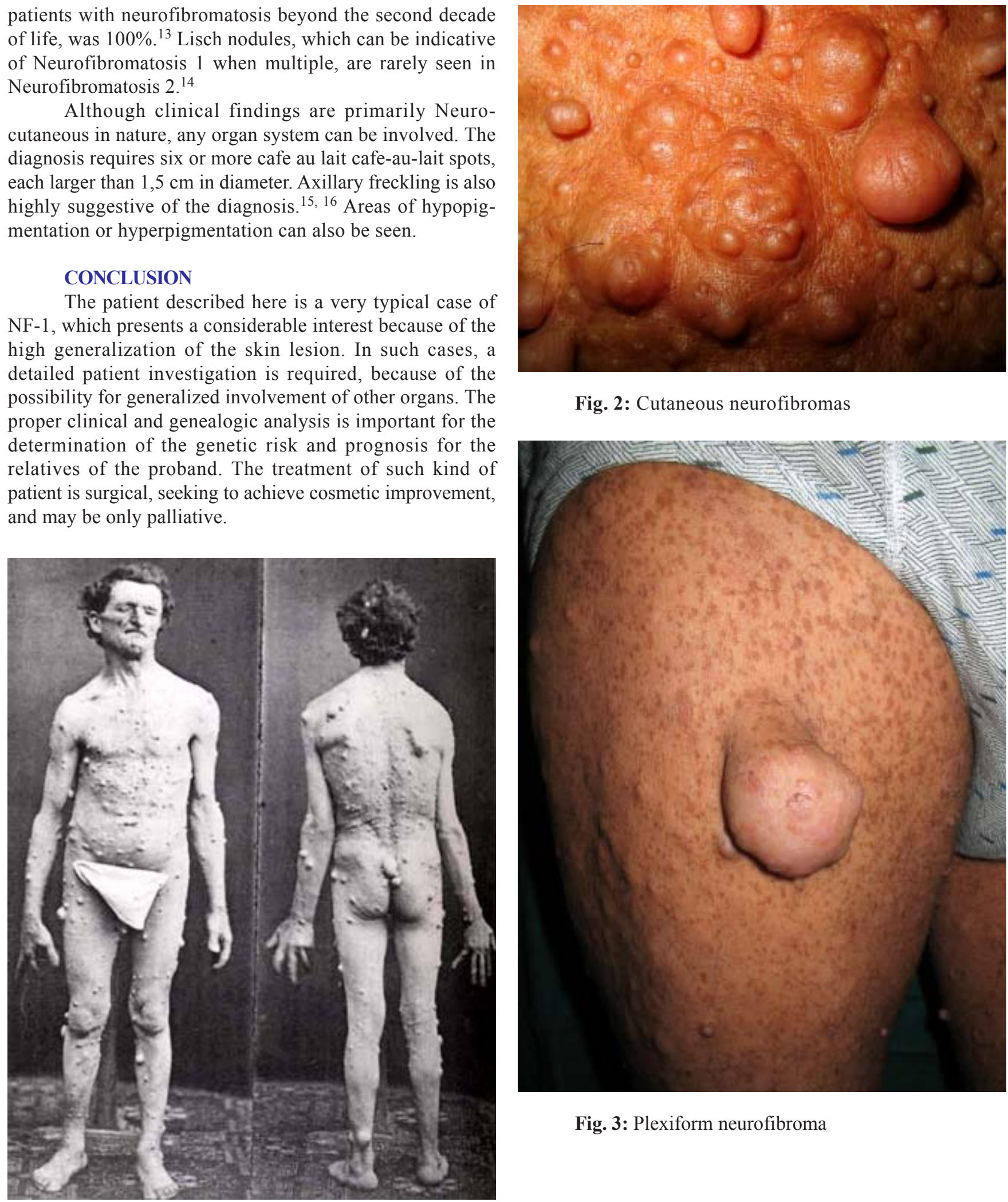

Fig. 2: Cutaneous neurofibromas

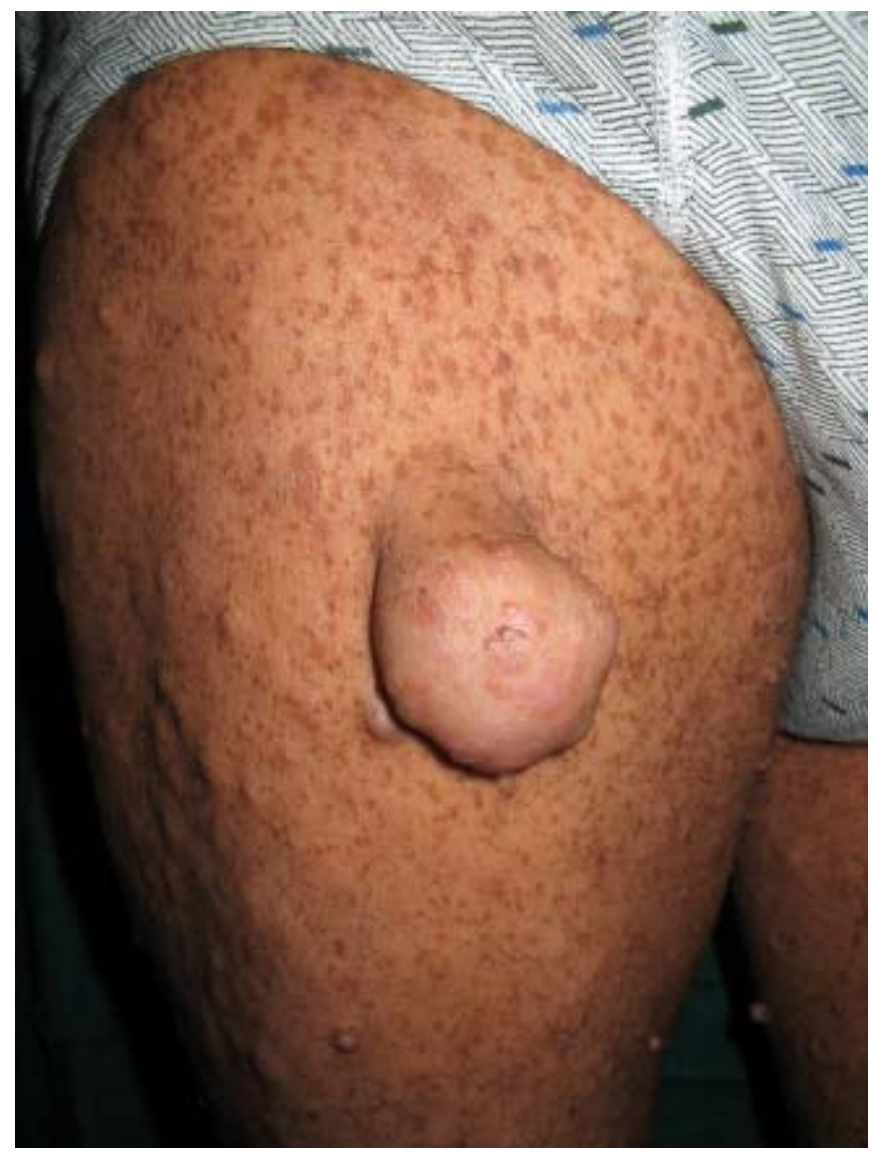

Fig. 3: Plexiform neurofibroma

Fig. 1: The patient original described from von Recklinghausen 


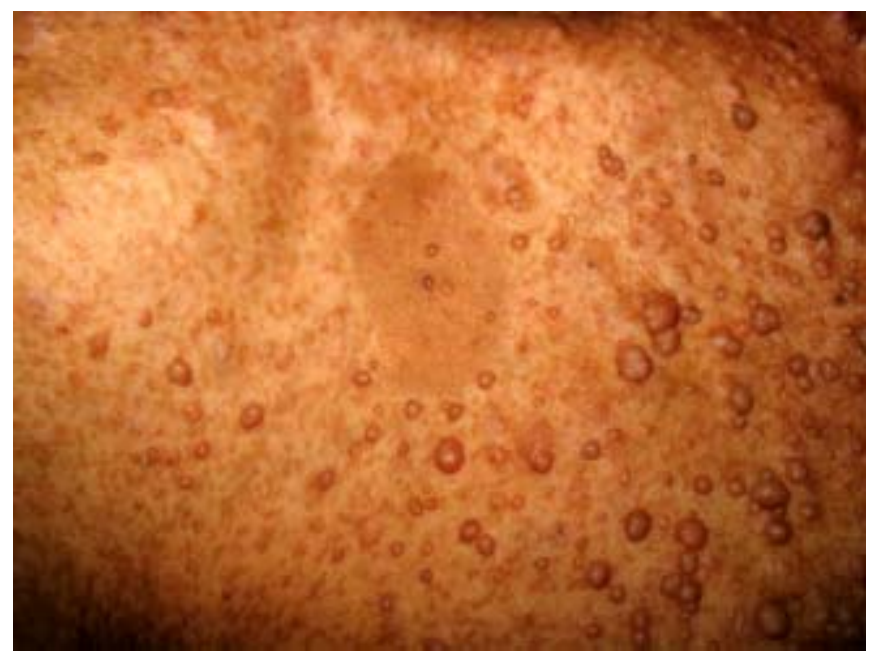

fig 4: Cafŭ-au-lait spot

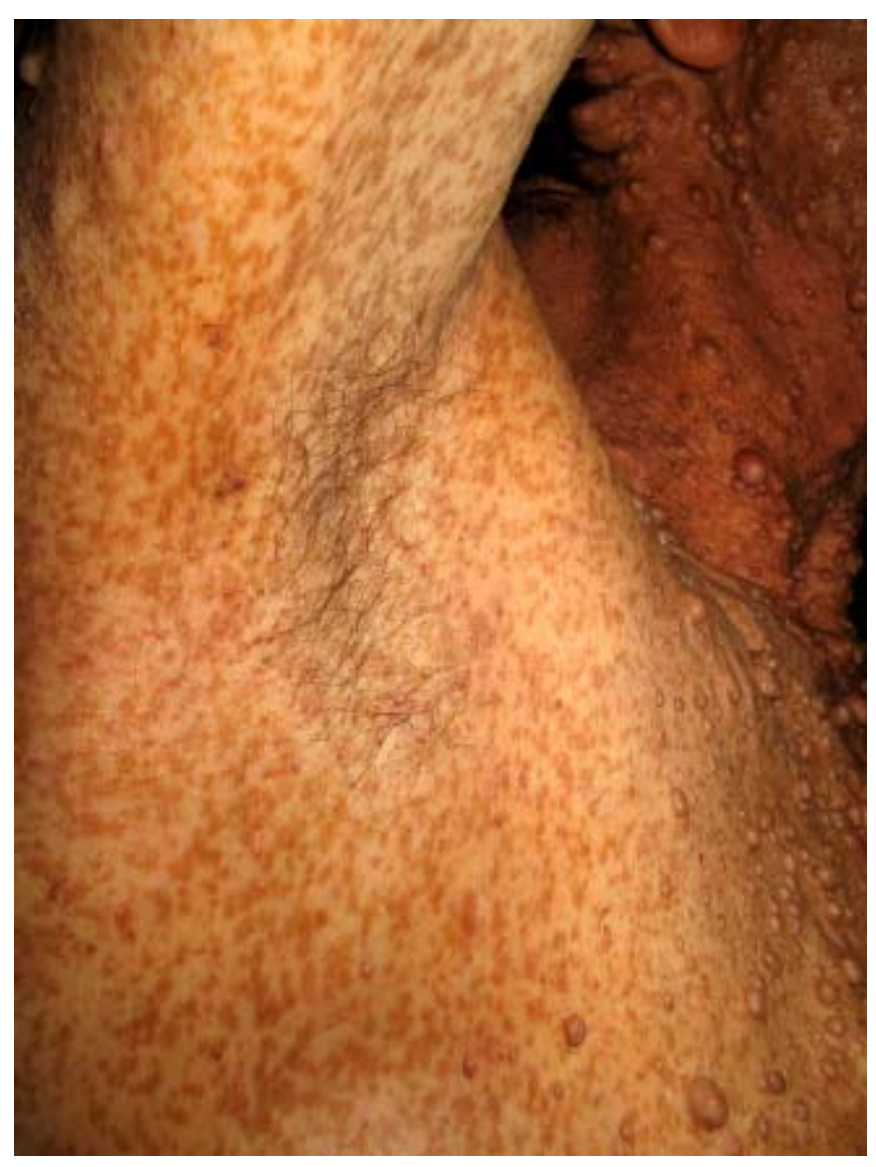

Fig. 5: Axillary freckling

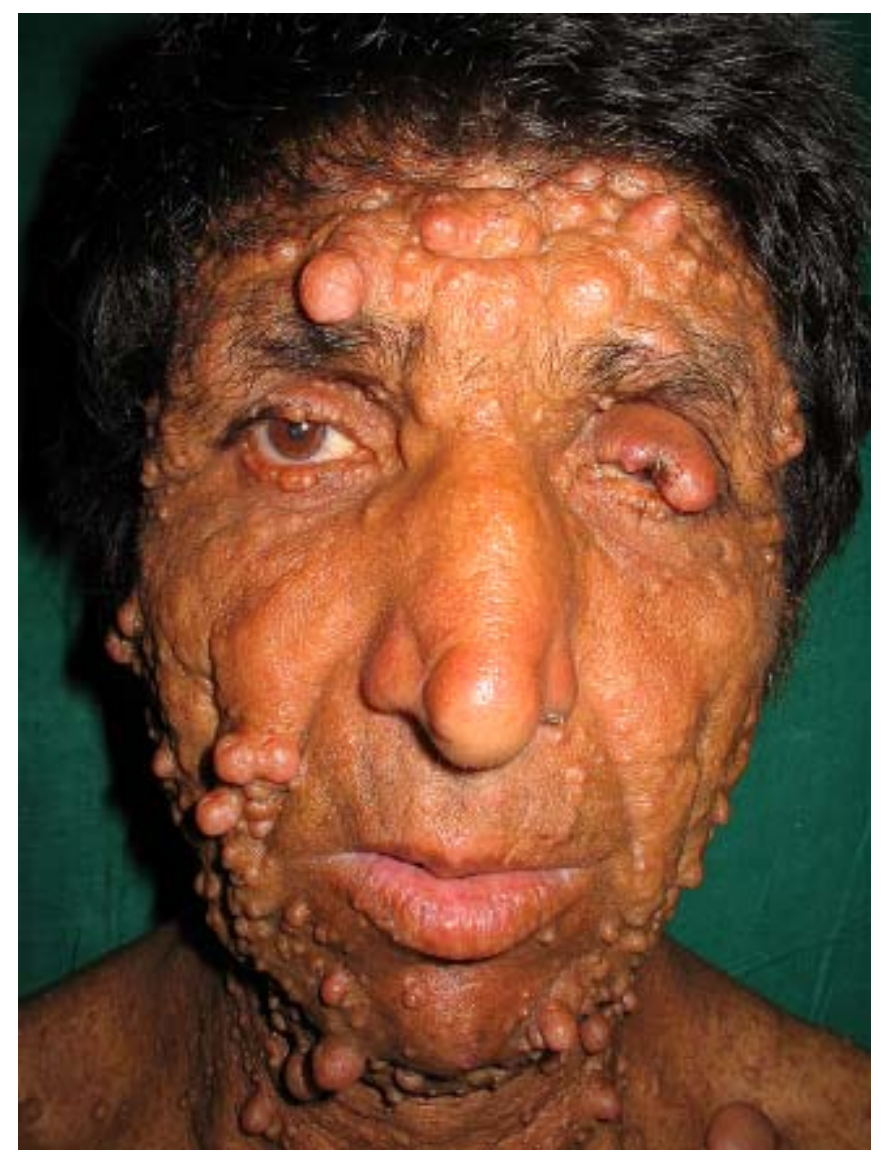

Fig. 6: Cutaneous fibromas on eyelids

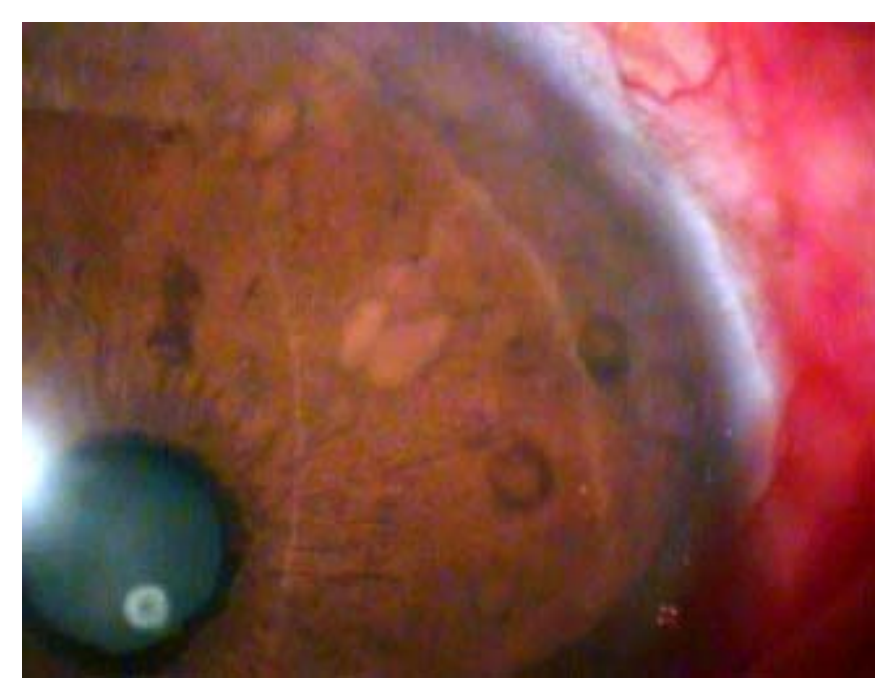

Fig 7: Lisch's nodules 


\section{BIBLIOGRAPHY}

1. Kobrin JL, Blodi FC, Weingeist TA: Ocular and orbital manifestations of neurofibromatosis. Surv Ophthalmol 24:45, 1979

2. von Recklinghausen FD: Ueber die multiplen Fibrome der Haut und ihre Beziehung zu den multiplen Neuromen. Berlin, Hirschwald, 1882

3. Riccardi VM: Neurofibromatosis: Clinical heterogeneity. Curr Probl Cancer $7: 1,1982$

4. Crowe FW, Schull WJ, Neel JV: A clinical, pathological, and genetic study of multiple neurofibromatosis. Springfield, IL, Charles C Thomas, 1956

5. Huson SM, Harper PS, Compston D: Von Recklinghausen neurofibromatosis: a clinical and population study in SouthEast Wales. Brain 111:1355, 1988

6. National Institutes of Health
Consensus Development Conference: Neurofibromatosis. Arch Neurol Chicago 45: 575,1988

7. Smith B, English FP: Classical eyelid border sign of neurofibromatosis. $\mathrm{Br} \mathrm{J}$ Ophthalmol 54:134, 1970

8. Riccardi VM, Eichner JE: Neurofibromatosis: phenotype, Natural History and Pathogenesis. Baltimore, Johns Hopkins University Press, 1986

9. Skuse GR, Kosciolek BA, Rowley PT: The neurofibroma in von Recklinghausen neurofibromatosis has a unicellular origin. Am J Hum Genet 49:600, 1991

10. Slater C, Hayes M, Saxe N et al: Macromelanosomes in the early diagnosis of neurofibromatosis. Am J Dermatopathol 8:284, 1986

11. Lewis RA, Riccardi VM: von
Recklinghausen neurofibromatosis: Incidence of iris hamartomata. Ophthalmology 88:348, 1981

12. Yanoff $M$, Fine BS: Ocular Pathology, 2nd ed. Philadelphia, Harper \& Row, 1982

13. Lubs ME, Bauer MS, Formas ME, Djokic B: Lisch nodules in neurofibromatosis type 1 . N Engl J Med 324:1264, 1991

14. Lubs ME, Bauer MS, Formas ME, Djokic B: Lisch nodules in neurofibromatosis type 1 . N Engl J Med 324:1264, 1991

15. Crowe FW: Axillary freckling as a diagnostic aid in neurofibromatosis. Ann Intern Med 61:1142, 1964

16. Smith DW: Recognizable Patterns of Human Malformation. Philadelphia, WB Saunders, 1982

\section{Address for correspondence:}

Dr. Valentina Dimitrova

Department of Dermatology and Venereology, Medical University of Pleven 91, Gen. Vladimir Vazov str., 5800 Pleven, Bulgaria

Tel./Fax: +359 64886 622;

E-mail: valentinadim@gmail.com 